\title{
TRAJECTORY TRACKING FOR A MOBILE ROBOT WITH SKID-SLIP COMPENSATION IN THE VECTOR-FIELD-ORIENTATION CONTROL SYSTEM
}

\author{
Maciej MiCHAŁEK, PIOTR DUTKIEWICZ, MARCin KIEŁCZEWSKI, DARIUSZ PAZDERSKI
}

\author{
Chair of Control and Systems Engineering \\ Poznań University of Technology, Piotrowo 3A, 60-965 Poznań, Poland \\ e-mail: \{Maciej.Michalek, Piotr.Dutkiewicz\}@put.poznan.pl \\ \{Marcin.Kielczewski, Dariusz.Pazderski\}@put.poznan.pl
}

\begin{abstract}
The article is devoted to a motion control problem for a differentially driven mobile robot in the task of trajectory tracking in the presence of skid-slip effects. The kinematic control concept presented in the paper is the Vector Field Orientation (VFO) feedback approach with a nonlinear feed-forward skid-slip influence compensation scheme. The VFO control law guarantees asymptotic convergence of the position tracking error to zero in spite of the disturbing influence of skid-slip phenomena. The paper includes a control law design description, stability and convergence analysis of a closed-loop system, and practical verification of the proposed control concept. The experimental results illustrate control quality obtained on a laboratory setup equipped with vision feedback, where the Kalman filter algorithm was used in order to practically estimate skid-slip components.
\end{abstract}

Keywords: differentially driven mobile robot, skid-slip compensation, trajectory tracking, vector fields.

\section{Introduction}

In recent years, due to the growing popularity and importance in many applications of Wheeled Mobile Robots (WMRs), motion control problems dedicated to WMRs attracted much attention in the robotics and automation community. Most wheeled vehicles can be regarded as mechanical systems with nonintegrable velocity (i.e., nonholonomic) constraints. Such simplification may be justified, at least locally, when the vehicle moves with relatively low velocities and accelerations on a firm and adhesive ground. In these motion conditions the dynamic effects, including wheel-ground interactions, can be regarded as a non significant and vanishing disturbance, and kinematic closed-loop control solutions are sufficient. However, in some real-life motion tasks, skid-slip phenomena cannot be longer ignored since they may substantially deteriorate motion accuracy of WMRs which are controlled using the assumption of nonholonomically constrained motion. One can easily find examples of motion conditions where nonholonomic constraints are violated, like motion on a sloping and slippery ground or losing adhesion between wheels and a motion surface when a vehicle rapidly changes the direction of motion. More- over, there exists an important group of vehicles equipped with a multi-wheel drive system known as Skid-Steering Wheeled Mobile Robots (SSWMRs), where skid and slip are intrinsic phenomena necessary for proper vehicle operation. For the latter class of underactuated systems, the set of feasible accelerations is restricted and their dynamics are not integrable (Lewis, 1999).

The issue of skid-slip modeling is a complicated problem, since it involves the description of forces acting between wheels and a motion surface based on highly nonlinear physical dynamic effects. Many works in this field were inspired by automotive industry, see, for example, (Pacejka, 2002; Kiencke and Nielsen, 2000; Wong, 2001). Some related works concentrate on improving motion stability and safety of car-like vehicles (Fukao et al., 2001), including anti-lock active drive systems (Mi et al., 2005). However, in the literature focused on robotics, not much has been written about solving fundamental control tasks for WMRs in the presence of slipping and skidding so far. Hence, the motion control problem for WMRs in skid-slip conditions still remains a challenge. Especially, there is a growing need for intensive experimental validation of the existing and new control strategies. Taking into account the skid-slip treat- 
ment for control purposes, one can notice two main alternative approaches. In the first one, the authors concentrate on skid-slip description at a dynamic level (Peng et al., 2004; Motte and Campion, 2000), also with respect to SSWMRs (Lhomme-Desages et al., 2007; Zong et al., 2006). Though dynamic modeling of skid-slip effects gives a theoretical possibility of obtaining the best accuracy and robustness of the control action, model complexity may in fact substantially complicate the overall control strategy and make its applicability relatively demanding. An alternative approach lies in a solely kinematic description of skid-slip phenomena (Wang and Low, 2008; Lenain et al., 2006; Corradini et al., 1999). It seems to be practically attractive (due to its simplicity) and also justified, since a direct consequence of skid-slip phenomena, regardless of their possible physical reason, is always a velocity disturbance on a kinematic level.

Recently, (Wang and Low, 2008) presented a classification of WMR kinematics from the perspective of the skid-slip influence on the kinematic model together with their controllability property. The work (Dixon et al., 2000) can be an example of the kinematic control approach, where the smooth control solution was designed based on the assumption that skid-slip disturbances are not measured and asymptotic error convergence may be relaxed leading to the practical stability result. Experimental verification of a similar control strategy for SSWMRs was presented in (Pazderski and Kozłowski, 2008). Other solutions, which combine both kinematic and dynamic approaches, have been also proposed, see, for example, (Leroquais and dAndrea Novel, 1996).

In this paper, we propose an extension of the VFO kinematic controller described in detail in (Michałek and Kozłowski, 2009) for a differentially driven mobile vehicle. The VFO controller is adopted here to the tracking task defined in the presence of limited skid-slip phenomena. The main idea proposed in the paper relies on treating skid-slip effects solely on a kinematic level and compensating their influence on the robot motion in a nonlinear feed-forward compensation loop. For practical estimation of skid-slip components, the velocity and acceleration observer in the form of a discrete Kalman filter using vision feedback measurement signals is proposed. Theoretical deliberations are experimentally verified on a laboratory setup with a differentially driven mobile robot for three different skid-slip motion conditions.

\section{Control task formulation}

The nominal model of differentially driven vehicle kinematics (simplified here to the unicycle) can be formulated as follows:

$$
\left[\begin{array}{c}
\dot{\theta} \\
\dot{x} \\
\dot{y}
\end{array}\right]=\left[\begin{array}{cc}
1 & 0 \\
0 & \cos \theta \\
0 & \sin \theta
\end{array}\right]\left[\begin{array}{l}
u_{1} \\
u_{2}
\end{array}\right] \Leftarrow \dot{\boldsymbol{q}}=\boldsymbol{G}(\boldsymbol{q}) \boldsymbol{u},
$$

where $\boldsymbol{q}=\left[\begin{array}{lll}\theta & x & y\end{array}\right]^{T} \in \mathbb{R}^{3}$ is a state vector describing the orientation and position coordinates of the vehicle platform in a global frame $\{X, Y\}$, respectively (see Fig. 10. The input vector $\boldsymbol{u}=\left[\begin{array}{ll}u_{1} & u_{2}\end{array}\right]^{T} \in \mathbb{R}^{2}$ consists of an angular and a longitudinal platform velocity, respectively, and $\dot{\boldsymbol{q}} \equiv \dot{\boldsymbol{q}}_{N}$ is a nominal state velocity. The expressions nominal model and nominal velocity mean here that (1) describes vehicle kinematics in the absence of external disturbances, especially skid-slip effects. It simultaneously implies that nonholonomic kinematic constraints, described in the Pfaffian form as $\boldsymbol{A}(\boldsymbol{q}) \dot{\boldsymbol{q}}=0$, $\boldsymbol{A}(\boldsymbol{q})=[0-\sin \theta \cos \theta]$, are satisfied.

Let us now define skid-slip phenomena in relation to the examined kinematics (1). Because the crucial principle of the VFO motion control approach presented in this paper relies solely on kinematic treatment of the unicycle vehicle model, the same should apply to skid and slip. Thus, we are not interested here in any physical reasons for skid-slip phenomena, since they are connected with hardly modeled friction effects between vehicle wheels and the motion surface. We are interested rather in the final results of skidding and slipping, which on the kinematic level always reduce to the additional velocity disturbance vector $\boldsymbol{v}_{s} \in \mathbb{R}^{3}$ called hereafter skid-slip velocity. The nominal model (1) is rewritten in this case in the disturbed form:

$$
\dot{\boldsymbol{q}}=\dot{\boldsymbol{q}}_{N}+\boldsymbol{v}_{s} \Rightarrow \quad \dot{\boldsymbol{q}}=\boldsymbol{g}_{1} u_{1}+\boldsymbol{g}_{2}(\boldsymbol{q}) u_{2}+\boldsymbol{v}_{s},
$$

where $\left[\boldsymbol{g}_{1} \boldsymbol{g}_{2}(\boldsymbol{q})\right] \equiv \boldsymbol{G}(\boldsymbol{q})$ and $\dot{\boldsymbol{q}}_{N}=\boldsymbol{G}(\boldsymbol{q}) \boldsymbol{u}$ (according to (1)), and $\boldsymbol{v}_{s}=\left[\begin{array}{lll}\omega_{s} & v_{s x} & v_{s y}\end{array}\right]^{T}=\left[\begin{array}{ll}\omega_{s} & \boldsymbol{v}_{s}^{* T}\end{array}\right]^{T}$ is the skidslip velocity expressed in a global frame with the angular component $\omega_{s} \in \mathbb{R}$ (yaw rate disturbance) and the driving component $\boldsymbol{v}_{s}^{*} \in \mathbb{R}^{2}$, respectively (cf. Fig. 1). Note that the general model (2) allows treating the term $\boldsymbol{v}_{s}$ as a resultant reduced disturbance describing, apart from the skid-slip influence, also other non-modeled effects like the dynamics of a vehicle platform or motor drives.

Let us now make the following three assumptions in relation to the disturbance $\boldsymbol{v}_{s}$ :

A1. The skid-slip velocity components and the skid-slip angle $\delta_{s}$ are bounded: $\omega_{s},\left\|\boldsymbol{v}_{s}^{*}\right\|, \delta_{s} \in \mathcal{L}_{\infty}$, where $\delta_{s} \triangleq \arg \left(\dot{\boldsymbol{q}}^{*}\right)-\arg \left(\dot{\boldsymbol{q}}_{N}^{*}\right)=\delta-\theta$ (see Fig. 1).

A2. The driving components of the skid-slip velocity are continuous: $\left\|\dot{\boldsymbol{v}}_{s}^{*}\right\| \in \mathcal{L}_{\infty}$.

A3. $\omega_{s}, \boldsymbol{v}_{s}^{*}$ and $\dot{\boldsymbol{v}}_{s}^{*}$ are measurable or can be estimated.

Assumption A1 is fundamental, since it preserves partial controllability of the system (2) from input $\boldsymbol{u}$ (see (Wang and Low, 2008)). The boundedness of the skid-slip angle $\delta_{s}$ is practically justified and means in this case that admissible skid-slip conditions exclude the persistent rotation of $\boldsymbol{v}_{s}^{*}$ vectorl. The boundedness of $\dot{\boldsymbol{v}}_{s}^{*}$ (A2) is in-

\footnotetext{
${ }^{1}$ The authors in (Wang and Low, 2008) postulate even more stringent assumption, namely, $\left|\delta_{s}\right|<\pi / 2$.
} 


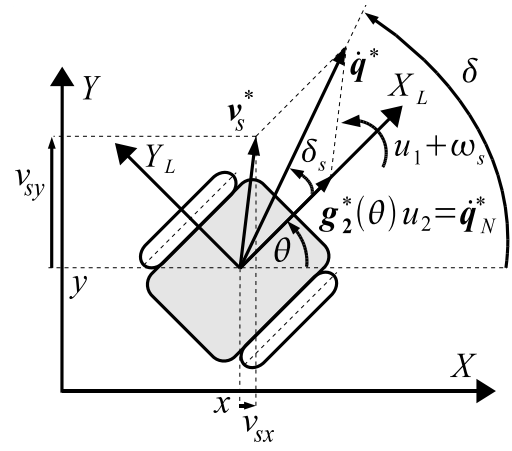

Fig. 1. Differentially driven vehicle in the presence of non-zero skid-slip velocity with the skid-slip angle $\delta_{s}$ denoted (notation: $\dot{\boldsymbol{q}}^{*}=[\dot{x} \dot{y}]^{T}, \boldsymbol{g}_{2}^{*}(\theta)=[\cos \theta \sin \theta]^{T}$ ).

volved by the proposed VFO control strategy to ensure the boundedness of control inputs in the closed-loop system, as will be explained in the sequel. Assumption A3 is required to physically realize the feed-forward compensation loop in the proposed control strategy. The latter issue will be commented in detail in Section 4

To formulate the main control task, let us assume that there is given the nominal reference trajectory $\boldsymbol{q}_{t}(\tau)=$ $\left[\theta_{t}(\tau) x_{t}(\tau) y_{t}(\tau)\right]^{T} \in \mathbb{R}^{3}$ which satisfies the equation (1) for some reference inputs $u_{1 t}(\tau)$ and $u_{2 t}(\tau)$ such that $\forall_{\tau \geqslant 0} u_{2 t}(\tau) \neq 0$. Define also the posture tracking error as follows:

$$
\boldsymbol{e}(\tau)=\left[\begin{array}{c}
e_{\theta}(\tau) \\
\boldsymbol{e}^{*}(\tau)
\end{array}\right] \triangleq\left[\begin{array}{c}
\theta_{t}(\tau)-\theta(\tau) \\
\boldsymbol{q}_{t}^{*}(\tau)-\boldsymbol{q}^{*}(\tau)
\end{array}\right] \in \mathbb{R}^{3}
$$

where $\boldsymbol{q}_{t}^{*}(\tau)=\left[x_{t}(\tau) y_{t}(\tau)\right]^{T}$ and $\boldsymbol{q}^{*}(\tau)=[x(\tau) y(\tau)]^{T}$. The main objective is to propose for the disturbed system (2) the feedback control law $\boldsymbol{u}=\boldsymbol{u}\left(\boldsymbol{q}_{t}, \boldsymbol{q}, \cdot\right)$, which ensures the stability of the closed-loop system with asymptotic convergence of the position error $e^{*}(\tau)$ to zero together with the boundedness of the orientation error $e_{\theta}(\tau)$ in the presence of a non-zero skid-slip velocity $\boldsymbol{v}_{s}$ meeting Assumptions A1-A3.

\section{VFO control strategy with skid-slip compensation}

The VFO approach originated from simple and intuitive geometrical interpretations related to nominal kinematics (1). A detailed description of the method and its application to classical control tasks were presented in (Michałek and Kozłowski, 2009). Due to the geometrical character of the VFO strategy and simple interpretations of particular control components, the original VFO concept can be adopted also to non-nominal motion conditions like in the case of the disturbed model (2). This adoption leads to the position tracking VFO controller with skid-slip influence compensation by a nonlinear feed-forward loop.
The control strategy together with the stability and convergence analysis of a closed-loop system will be described below. For brevity, the explanatory description will be restricted only to the most important issues which will allow understanding the motivation of particular control components. For more detailed deliberations on VFO methodology, the reader is referred to (Michałek and Kozłowski, 2009) and also to the preliminary work (Michałek, 2007).

To make our description clear, let us first decompose the system (2) into two subsystems:

$$
\begin{aligned}
\dot{\theta} & =u_{1}+\omega_{s}, \\
\dot{\boldsymbol{q}}^{*} & =\boldsymbol{g}_{2}^{*}(\theta) u_{2}+\boldsymbol{v}_{s}^{*}=\dot{\boldsymbol{q}}_{N}^{*}+\boldsymbol{v}_{s}^{*},
\end{aligned}
$$

where

$$
\dot{\boldsymbol{q}}^{*}=\left[\begin{array}{c}
\dot{x} \\
\dot{y}
\end{array}\right], \quad \boldsymbol{g}_{2}^{*}(\theta)=\left[\begin{array}{c}
\cos \theta \\
\sin \theta
\end{array}\right], \quad \boldsymbol{v}_{s}^{*}=\left[\begin{array}{c}
v_{s x} \\
v_{s y}
\end{array}\right] .
$$

One of the crucial elements of the VFO strategy is the socalled convergence vector field,

$$
\boldsymbol{h}=\left[\begin{array}{l}
h_{1} \\
h_{2} \\
h_{3}
\end{array}\right]=\left[\begin{array}{l}
h_{1} \\
\boldsymbol{h}^{*}
\end{array}\right] \in \mathbb{R}^{3}, \quad \boldsymbol{h}^{*} \in \mathbb{R}^{2} .
$$

This vector field determines at every time instant the field of velocity vectors in the vehicle state space. Assume that at every point $\boldsymbol{q}$ of the vehicle state space $\boldsymbol{h}^{*}\left(\boldsymbol{q}, \boldsymbol{q}_{t}, \cdot\right)$ defines the instantaneous 2 desired direction, orientation (connected with the vector sense) and some kind of distance to the reference position trajectory $\boldsymbol{q}_{t}^{*}=\left[x_{t} y_{t}\right]^{T}$. Simultaneously, the first component, $h_{1}$, determines the desired behavior for the first state variable $\theta$. According to the above description, one can say that $\boldsymbol{h}$ defines the desired instantaneous evolution for the controlled vehicle in the state space. Hence, to accomplish the control task, it seems sufficient to make the instantaneous vehicle velocity $\dot{\boldsymbol{q}}$ converge (at least at the limit) to the current vector $\boldsymbol{h}$, which can be written as

$$
\lim _{\tau \rightarrow \infty}\left[\dot{\boldsymbol{q}}(\tau)-\boldsymbol{h}\left(\boldsymbol{q}(\tau), \boldsymbol{q}_{t}(\tau), \cdot\right)\right]=\mathbf{0} .
$$

Substituting to the above limit appropriate components from (4), (5) and (7), one can rewrite it into the following form:

$$
\lim _{\tau \rightarrow \infty}\left\{\begin{array}{l}
u_{1}(\tau)+\omega_{s}(\tau)-h_{1}(\tau)=0 \\
u_{2}(\tau) \cos \theta(\tau)+v_{s x}(\tau)-h_{2}(\tau)=0 \\
u_{2}(\tau) \sin \theta(\tau)+v_{s y}(\tau)-h_{3}(\tau)=0
\end{array}\right.
$$

The first of the above relations can be met instantaneously (omitting the limit) defining the first control input as

$$
u_{1}(\tau) \triangleq h_{1}(\tau)-\omega_{s}(\tau) .
$$

\footnotetext{
${ }^{2}$ For the trajectory tracking task, the convergence vector field is timevarying.
} 
On the other hand, the last two relations from (9) can be combined in a more compact form yielding the so-called orienting condition?

$$
\lim _{\tau \rightarrow \infty}\left[\operatorname{Atan} 2\left(H_{3} \operatorname{sgn}\left(u_{2}\right), H_{2} \operatorname{sgn}\left(u_{2}\right)\right)-\theta(\tau)\right]=0,
$$

where

$$
\boldsymbol{H}^{*}=\left[\begin{array}{c}
H_{2} \\
H_{3}
\end{array}\right]=\left[\begin{array}{l}
h_{2}-v_{s x} \\
h_{3}-v_{s y}
\end{array}\right]=\boldsymbol{h}^{*}-\boldsymbol{v}_{s}^{*} .
$$

The condition (11) cannot generally be satisfied instantaneously due to the integral relation between the variable $\theta$ and the input $u_{1}$ (see (4)). Thus, let us introduce the auxiliary variable

$$
\theta_{a}(\tau) \triangleq \operatorname{Atan} 2 \mathrm{c}\left(H_{3} \operatorname{sgn}\left(u_{2 t}\right), H_{2} \operatorname{sgn}\left(u_{2 t}\right)\right) \in \mathbb{R},
$$

where $\operatorname{Atan} 2 \mathrm{c}(\cdot, \cdot): \mathbb{R} \times \mathbb{R} \mapsto \mathbb{R}$ is a continuous version of the function $\operatorname{Atan} 2(\cdot, \cdot): \mathbb{R} \times \mathbb{R} \mapsto(-\pi, \pi]$. In the definition $(13)$, the term $\operatorname{sgn}\left(u_{2}\right)$ was replaced with $\operatorname{sgn}\left(u_{2 t}\right)$ in order to guarantee smooth transient behavior of a vehicle in forward as well as backward reference motion 4

Now, to meet the relation 11, it suffices to make the auxiliary error

$$
e_{a}(\tau) \triangleq \theta_{a}(\tau)-\theta(\tau), \quad e_{a}(\tau) \in \mathbb{R},
$$

tend to zero. This can be accomplished by proper definition of the first component of $\boldsymbol{h}$, since it determines the desired behavior for the $\theta$ state variable and, connected with the proposition (10), will give the final definition for the input $u_{1}$.

The components of $\boldsymbol{h}$ can be defined in several ways. One possible proposition is as follows:

$$
\begin{aligned}
& h_{1}(\tau) \triangleq k_{1} e_{a}(\tau)+\dot{\theta}_{a}(\tau), \\
& \boldsymbol{h}^{*}(\tau) \triangleq k_{p} \boldsymbol{e}^{*}(\tau)+\dot{\boldsymbol{q}}_{t}^{*}(\tau),
\end{aligned}
$$

where $k_{1}, k_{p}>0$ are the design coefficients, $\dot{\boldsymbol{q}}_{t}^{*}(\tau)=$ $\left[\dot{x}_{t}(\tau) \dot{y}_{t}(\tau)\right]^{T}$ is the reference longitudinal velocity vector, and the feed-forward term

$$
\dot{\theta}_{a}(\tau)=\frac{\dot{H}_{3}(\tau) H_{2}(\tau)-H_{3}(\tau) \dot{H}_{2}(\tau)}{\left\|\boldsymbol{H}^{*}(\tau)\right\|^{2}}
$$

results from the time-differentiation of (13). Now one can determine all the terms needed to compute the first control input $u_{1}$ defined in (10).

It remains now to define the second control input $u_{2}$. According to the VFO strategy, the input $u_{2}$ is called pushing control, since in geometrical interpretation it pushes

\footnotetext{
${ }^{3}$ The terminology comes from the VFO methodology, see (Michałek and Kozłowski, 2009).

${ }^{4}$ The value of $\operatorname{sgn}\left(u_{2 t}\right) \in\{-1,+1\}$ remains constant during a tracking task since $\forall_{\tau \geqslant 0} u_{2 t}(\tau) \neq 0$ according to the assumption made in Section 2
}

the substate $q^{*}$ in the subsystem (5) along the current direction of $\boldsymbol{g}_{2}^{*}(\theta)$. From (5) and (7) it can be seen that, to meet (8), the value of $u_{2}$ should guarantee that the norm of $\dot{\boldsymbol{q}}^{*}$ is convergent to the norm of the $\boldsymbol{h}^{*}$ vector (at least at the limit). It is equivalent to the convergence of the nominal velocity $\dot{\boldsymbol{q}}_{N}^{*}$ to $\boldsymbol{H}^{*}$ (see (12)). Using the heuristic careful pushing strategy, the second input is defined as follows:

$$
u_{2}(\tau) \triangleq\left\|\boldsymbol{H}^{*}(\tau)\right\| \cos \alpha(\tau)=\boldsymbol{g}_{2}^{* T}(\theta(\tau)) \boldsymbol{H}^{*}(\tau),
$$

where $\alpha(\tau)=\angle\left(\boldsymbol{g}_{2}^{*}(\theta(\tau)), \boldsymbol{H}^{*}(\tau)\right)$. The definition 18 admits pushing with intensity proportional to the current orthogonal projection of $\boldsymbol{H}^{*}$ onto a direction of the nominal longitudinal velocity $\dot{\boldsymbol{q}}_{N}^{*}$ determined by $\boldsymbol{g}_{2}^{*}(\theta)$ (cf. (5)). The maximal pushing intensity is thus possible only when directions of $\boldsymbol{g}_{2}^{*}(\theta)$ and $\boldsymbol{H}^{*}$ are perfectly matched $(|\cos \alpha|=1)$, which in turn depends on the control action effectiveness of the input $u_{1}$.

Summarizing, one can say that the whole feedback control process is divided into two subprocessesorienting and pushing - connected with the control inputs $u_{1}$ and $u_{2}$, respectively. The orienting control $u_{1}$ is responsible for driving the vehicle orientation $\theta$ toward the auxiliary variable $\theta_{a}$, which in turn determines the direction of the $\boldsymbol{H}^{*}$ vector on a motion plane (see (13)). On the other hand, the $u_{2}$ control input is responsible for driving the vehicle with a proper intensity proportional to the instantaneous norm of the $\boldsymbol{H}^{*}$ vector (in the careful pushing strategy). Both control inputs cooperate for the desired final effect where the influence of skid-slip phenomena on the vehicle motion is compensated and, as a consequence, the resultant longitudinal velocity $\dot{q}^{*}$ perfectly matches the instantaneous convergence vector $\boldsymbol{h}^{*}$, ensuring asymptotic convergence of the vehicle position $\boldsymbol{q}^{*}$ to the reference one $\boldsymbol{q}_{t}^{*}$. The control concept is also explained in Fig. 2, where the ideal case of perfect skid-slip compensation and perfect matching for $\dot{\boldsymbol{q}}^{*}$ with $\boldsymbol{h}^{*}$ is presented. Note that the control objective is not the elimination of skid-slip phenomena, but rather the compensation of their influence on vehicle position evolution. Collecting the definitions (10) and (18) with particular terms from (15) and (6) finally allows presenting the VFO control law with skid-slip compensation as follows:

$$
\begin{aligned}
& u_{1}(\tau):=k_{1} e_{a}(\tau)+\dot{\theta}_{a}(\tau)-\omega_{s}(\tau), \\
& u_{2}(\tau):=H_{2}(\tau) \cos \theta(\tau)+H_{3}(\tau) \sin \theta(\tau),
\end{aligned}
$$

with $\mathrm{H}_{2}$ and $\mathrm{H}_{3}$ introduced in (12). It is worth noting that the VFO strategy (19)-20) differs from the nominal VFO control law presented in (Michałek and Kozłowski, 2009) by the introduction of nonlinear feed-forward compensation of the skid-slip components $\omega_{s}, v_{s x}$ and $v_{s y}$. It means that simply zeroing the latter leads to the VFO controller in its nominal form dedicated for a tracking task in the absence of skid-slip effects. 


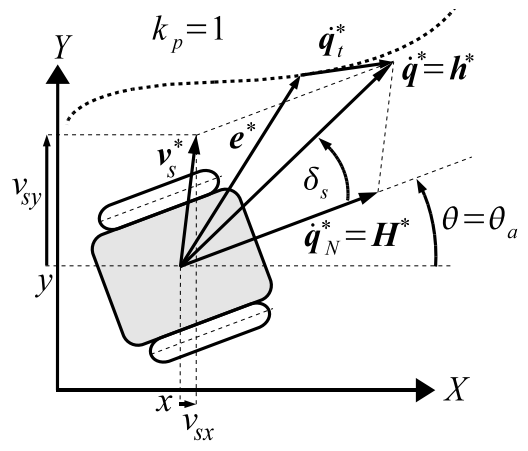

Fig. 2. VFO control strategy with skid-slip influence compensation for the ideal case of perfect matching between the vehicle driving velocity vector $\dot{\boldsymbol{q}}^{*}$ and the current convergence vector $\boldsymbol{h}^{*}$.

Let us now formulate the following proposition.

Proposition 1. Assuming that the reference trajectory $\boldsymbol{q}_{t}^{*}(\tau)$ is sufficiently smooth, $\left\|\dot{\boldsymbol{q}}_{t}^{*}\right\|,\left\|\ddot{\boldsymbol{q}}_{t}^{*}\right\| \in \mathcal{L}_{\infty}$, the skid-slip velocity $\boldsymbol{v}_{s}$ in (2) satisfies A1-A3, and that for all $\tau \geqslant 0$ there holds $\left\|\boldsymbol{H}^{*}(\tau)\right\| \neq 0$, the VFO control law (19), (20) applied to the system (21) guarantees the stability of the closed-loop system and asymptotic convergence of the position error $e^{*}(\tau)$ in the sense that $\left\|e^{*}(\tau)\right\|, e_{\theta}(\tau) \in \mathcal{L}_{\infty}$ and $\lim _{\tau \rightarrow \infty}\left\|e^{*}(\tau)\right\|=0$.

Proof. The formal analysis conducted below is an extended and detailed version of the proof presented in (Michałek, 2007).

Consider first time-evolution of the auxiliary error $e_{a}(\tau)$. Substituting (19) into (4) yields

$$
\dot{e}_{a}(\tau)+k_{1} e_{a}(\tau)=0 \Rightarrow \lim _{\tau \rightarrow \infty} e_{a}(\tau)=0,
$$

which shows the boundedness of $e_{a}(\tau)$ and exponential convergence of the $\theta$ state variable to the auxiliary variable $\theta_{a}$. This result will be useful in the subsequent analysis.

In a second step, let us consider the behavior of the position tracking error $e^{*}(\tau)$. Using the definitions (16) and (12), one can write

$$
\boldsymbol{H}^{*}=\boldsymbol{h}^{*}-\boldsymbol{v}_{s}^{*} \stackrel{\underline{16}}{\Longrightarrow} \boldsymbol{H}^{*}=k_{p} \boldsymbol{e}^{*}+\dot{\boldsymbol{q}}_{t}^{*}-\boldsymbol{v}_{s}^{*} .
$$

As a consequence of the fact that $\dot{\boldsymbol{e}}^{*}=\dot{\boldsymbol{q}}_{t}^{*}-\dot{\boldsymbol{q}}^{*}$, using (5) and substituting $\dot{\boldsymbol{q}}_{t}^{*}$ term taken from (22) one obtains the following equation:

$$
\dot{e}^{*}=-k_{p} \boldsymbol{e}^{*}+\boldsymbol{r}, \quad \text { where } \quad \boldsymbol{r}=\boldsymbol{H}^{*}-\boldsymbol{g}_{2}^{*}(\theta) u_{2} .
$$

It can be shown (see Appendix) that the following relations hold:

$$
\|\boldsymbol{r}\|=\left\|\boldsymbol{H}^{*}\right\| \gamma(\theta) \quad \text { and } \quad \lim _{\theta \rightarrow \theta_{a}} \gamma(\theta)=0,
$$

where $\gamma(\theta)=\sqrt{1-\cos ^{2} \alpha(\theta)} \in[0,1]$ and $\alpha(\theta)=$ $\angle\left(\boldsymbol{g}_{2}^{*}(\theta), \boldsymbol{H}^{*}\right)$. Introducing a positive definite function
$V\left(e^{*}\right)=\frac{1}{2} e^{* T} e^{*}$ and utilizing (23) and (24), one can estimate the time-derivative $\dot{V}\left(e^{*}\right)$ as follows:

$$
\begin{aligned}
\dot{V} & =\boldsymbol{e}^{* T} \dot{\boldsymbol{e}}^{*}=\boldsymbol{e}^{* T}\left(-k_{p} \boldsymbol{e}^{*}+\boldsymbol{r}\right) \\
& =-k_{p}\left\|\boldsymbol{e}^{*}\right\|^{2}+\boldsymbol{e}^{* T} \boldsymbol{r} \\
& \leqslant-k_{p}\left\|\boldsymbol{e}^{*}\right\|^{2}+\left\|\boldsymbol{e}^{*}\right\|\|\boldsymbol{r}\| \\
& =-k_{p}\left\|\boldsymbol{e}^{*}\right\|^{2}+\left\|\boldsymbol{e}^{*}\right\|\left\|\boldsymbol{H}^{*}\right\| \gamma \\
& =-k_{p}\left\|\boldsymbol{e}^{*}\right\|^{2}+\left\|\boldsymbol{e}^{*}\right\|\left\|k_{p} \boldsymbol{e}^{*}+\dot{\boldsymbol{q}}_{t}^{*}-\boldsymbol{v}_{s}^{*}\right\| \gamma \\
& \leqslant-k_{p}(1-\gamma)\left\|\boldsymbol{e}^{*}\right\|^{2}+\gamma\left\|\boldsymbol{e}^{*}\right\| \kappa=-W\left(\boldsymbol{e}^{*}, \gamma, \kappa\right),
\end{aligned}
$$

where $\kappa=\left\|\dot{\boldsymbol{q}}_{t}^{*}\right\|+\left\|\boldsymbol{v}_{s}^{*}\right\|$ is bounded by assumption and

$$
W\left(\boldsymbol{e}^{*}, \gamma, \kappa\right)=k_{p}(1-\gamma)\left\|\boldsymbol{e}^{*}\right\|^{2}-\gamma\left\|\boldsymbol{e}^{*}\right\| \kappa .
$$

The function (25) is positive if the following condition is met:

$$
\left\|\boldsymbol{e}^{*}(\tau)\right\|>\Gamma(\tau), \quad \Gamma(\tau)=\frac{\gamma(\tau) \kappa(\tau)}{k_{p}(1-\gamma(\tau))} .
$$

Let us analyze the above partial result using the following reasoning: The function $\Gamma$ from (26) is finite for $\gamma<1$. The case when $\gamma=1$ may appear, but is temporary and non-attractive (as a direct consequence of the limit in (24) together with the convergence result obtained in (21)). It can potentially occur only during a transient stage for some time instant $\bar{\tau}<\infty$. In this case, we have $W(\bar{\tau})=-\left\|\boldsymbol{e}^{*}(\bar{\tau})\right\| \kappa(\bar{\tau})<\infty$ (we assume that $\left.\left\|\boldsymbol{e}^{*}(0)\right\|<\infty\right)$, which implies that $\dot{V}(\bar{\tau})<\infty$. Hence, in the case when $\gamma=1$, the finite time escape for $\left\|e^{*}\right\|$ does not hold.

For all $\tau \neq \bar{\tau}$ the function $\Gamma(\tau)$ is finite and the norm $\left\|\boldsymbol{e}^{*}(\tau)\right\|$ is convergent if it becomes greater than $\Gamma(\tau)$. Since $\gamma(\tau)$ can never get stuck in $\gamma=1$ and, moreover, $\exists_{\tau_{\gamma}<\infty}: \forall_{\tau>\tau_{\gamma}} \gamma(\tau)<1$ (due to the similar reasons mentioned above) and since $\kappa \in \mathcal{L}_{\infty}$ (by assumption), one can conclude that (26) is determined for bounded $\Gamma(\tau)$ almost always and for all $\tau>\tau_{\gamma}$. As a consequence, $\left\|e^{*}(\tau)\right\| \in$ $\mathcal{L}_{\infty}$ and $V\left(e^{*}\right), \dot{V}\left(e^{*}\right) \in \mathcal{L}_{\infty}$. According to (25), (22) and (23), also $W,\left\|\boldsymbol{H}^{*}\right\|,\|\boldsymbol{r}\| \in \mathcal{L}_{\infty}$. Now, from the left-hand side of (23) one concludes that $\left\|\dot{e}^{*}\right\| \in \mathcal{L}_{\infty}$. Since the norms $\left\|e^{*}\right\|$ and $\left\|\dot{e}^{*}\right\|$ are bounded, one can utilize Barbalat's lemma in the formulation presented in Appendix. It can be shown that the function $W$ given by (25) is uniformly continuous and integrable (see Appendix), $W(\tau), \dot{W}(\tau) \in \mathcal{L}_{\infty}, \lim _{\tau \rightarrow \infty} \int_{0}^{\tau} W(\xi) d \xi<$ $\infty$. According to Barbalat's lemma, one can conclude that $\lim _{\tau \rightarrow \infty} W(\tau)=0$. It is equivalent (see (25) and (26) ) to $\lim _{\tau \rightarrow \infty}\left[\boldsymbol{e}^{*}(\tau)-\Gamma(\tau)\right]=0$. Recalling now that $\lim _{\tau \rightarrow \infty} \gamma(\tau)=0$ (see the limit from (24) together with (21)), one may conclude (see (26) that $\lim _{\tau \rightarrow \infty} \Gamma(\tau)=0$ and, finally, according to the preceding reasoning, $\lim _{\tau \rightarrow \infty}\left\|e^{*}(\tau)\right\|=0$.

Using the notation $\theta_{t}=\arg \left(\dot{\boldsymbol{q}}_{t}^{*}\right), \theta=\arg \left(\dot{\boldsymbol{q}}_{N}^{*}\right)$ (see (5)) together with the definition of the skid-slip angle as in 
Assumption A1, the boundedness of the $e_{\theta}(\tau)$ error can be concluded from the following reasoning. At the limit for $\tau \rightarrow \infty$, we have $\theta=\theta_{a}$ and $\left\|e^{*}\right\|=0$ and, as a direct consequence, $\boldsymbol{h}^{*}=\dot{\boldsymbol{q}}_{t}^{*}$ (see (16)) and $\dot{\boldsymbol{q}}^{*}=\boldsymbol{h}^{*}=\dot{\boldsymbol{q}}_{t}^{*}$. Proceeding, one obtains $\delta_{s}=\arg \left(\dot{\boldsymbol{q}}_{t}^{*}\right)-\arg \left(\dot{\boldsymbol{q}}_{N}^{*}\right)=\theta_{t}-$ $\theta=e_{\theta}$ (compare Fig. 2), which, according to A1, allows concluding $e_{\theta} \in \mathcal{L}_{\infty}$.

The assumption about the norm of $\boldsymbol{H}^{*}$ made in Proposition 1 involves a comment. The reason for its introduction comes from the definition (13) and its timederivative (17), which are not defined for $\left\|\boldsymbol{H}^{*}\right\|=0$. This fact generally makes the VFO law (19)-20) a discontinuous controller. Additional analysis reveals, however, that occasional violation of the above assumption does not have to relax the convergence result obtained in the proof. The equality $\left\|\boldsymbol{H}^{*}\right\|=0$ concerns two cases (compare (22), (23) and (16)):

C1. $e^{*} \neq 0 \wedge v_{s}^{*}=h^{*}$ (it may happen only during a transient stage)

C2. $\boldsymbol{e}^{*}=\mathbf{0} \wedge \boldsymbol{v}_{s}^{*}=\dot{\boldsymbol{q}}_{t}^{*}$ (when a system evolves exactly along a reference position trajectory $\boldsymbol{q}_{t}^{*}$ ).

Since for $\left\|\boldsymbol{H}^{*}\right\|=0$ also $u_{2}=0$ (see (20)), the only term which drives the subsystem (5) is the skid-slip velocity $\boldsymbol{v}_{s}^{*}$. As a consequence, Cases $\mathrm{C} 1$ and $\mathrm{C} 2$ describe a situation where the disturbance $\boldsymbol{v}_{s}^{*}$ drives the subsystem (5) toward $5 \boldsymbol{q}_{t}^{*}(\mathrm{C} 1)$ or along $\boldsymbol{q}_{t}^{*}(\mathrm{C} 2)$, satisfying the main control objective. Moreover, in Case $\mathrm{C} 1$ one obtains (see (23) $), \boldsymbol{r}=\mathbf{0} \Rightarrow \dot{\boldsymbol{e}}^{*}+k_{p} \boldsymbol{e}^{*}=\mathbf{0}$. Hence the boundedness and asymptotic convergence of $e^{*}$ are still preserved. It is important that the control discontinuity set determined by $\left\|\boldsymbol{H}^{*}\right\|=0$ is non-attractive and nonpersistent.

However, to obtain a well-defined control $u_{1}$ for the whole domain of its arguments, one can introduce additional definitions for the $\theta_{a}$ and $\dot{\theta}_{a}$ components in the assumed small $\epsilon$-vicinity of the point $\boldsymbol{H}^{*}=\mathbf{0}$. Let us propose to take

$$
\left\{\begin{array}{l}
\theta_{a}(\tau) \triangleq \theta_{a}\left(\tau_{-}\right) \\
\dot{\theta}_{a}(\tau) \triangleq 0
\end{array} \quad \text { for } \quad\left\|\boldsymbol{H}^{*}(\tau)\right\| \leqslant \epsilon,\right.
$$

where $0<\epsilon<\inf _{\tau}\left\|\dot{\boldsymbol{q}}_{t}^{*}(\tau)-\boldsymbol{v}_{s}^{*}(\tau)\right\|$, and $\tau_{-}$denotes the time instant when the norm $\left\|\boldsymbol{H}^{*}(\tau)\right\|$ reaches the $\epsilon$ vicinity. The proposed additional definitions together with (13) allow the control formula (19) to remain unchanged.

\section{Experimental verification}

4.1. Experimental setup. The experiments were conducted in the experimental setup presented schematically in Fig. 3. It consists of four main parts: a differentially

\footnotetext{
${ }^{5}$ Let us recall that $\boldsymbol{h}^{*}$ is an instantaneous convergence vector.
}

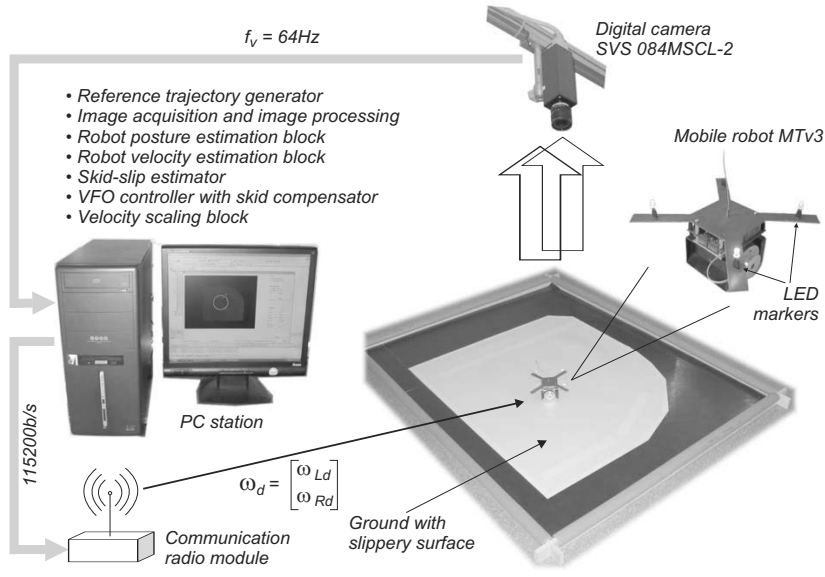

Fig. 3. Scheme of the experimental setup with the MTv3 mobile robot and the vision measurement system.

driven mobile robot MTv3, a flat test ground with a slippery surface, a digital vision system playing the role of a feedback sensor and allowing estimating particular components of robot state velocity, and a PC station with a communication radio module. The vision system works with a frequency of $f_{v}=64 \mathrm{~Hz}$. The localization of the mobile robot is possible due to three LED markers which are detected by the vision system and mounted on a special support on the top of the robot platform. The whole control system is designed in a cascaded form, where the PC computer plays the role of a supervisory layer computing VFO control signals and then sending the rescaled wheel velocities $\omega_{L d}, \omega_{R d}$ as command values to two PI board controllers, implemented on the mobile robot. The main computational procedures of the PC station are denoted in Fig. 3. Two of them, namely, Robot velocity estimation block and Skid-slip estimator, which are crucial for practical implementation of the proposed control approach, are described in the next subsection.

4.2. Practical estimation of skid-slip components. The main challenge in practical implementation of the control law (19)-20) comes from the need for effective estimation of skid-slip components. Apart from the velocity terms $\omega_{s}, v_{s x}$ and $v_{s y}$ needed in (19) and (12), also skid-slip accelerations are required in VFO computations because they appear in the components of the timederivative $\dot{\boldsymbol{H}}^{*}=k_{p} \dot{\boldsymbol{e}}^{*}+\ddot{\boldsymbol{q}}_{t}^{*}-\dot{\boldsymbol{v}}_{s}^{*}$ in Eqn. (17). Since the VFO control concept is a strictly kinematic approach, the solution to the estimation problem was formulated and implemented also in this manner.

In the kinematic approach, skid-slip velocities can be computed as a difference between the state velocity $\dot{\boldsymbol{q}}$ and the nominal velocity vector $\dot{\boldsymbol{q}}_{N}$ using the model equations (4) and (5):

$\omega_{s}=\dot{\theta}-u_{1}, \quad v_{s x}=\dot{x}-u_{2} \mathrm{c} \theta, \quad v_{s y}=\dot{y}-u_{2} \mathrm{~s} \theta$, 
with the notion $\mathrm{s} \theta \equiv \sin \theta, \mathrm{c} \theta \equiv \cos \theta$ used for compactness. The skid-slip accelerations involved in 17 can be obtained by formal time-differentiation of the latter two from the above equations yielding

$$
\dot{v}_{s x}=\ddot{x}+u_{2} \dot{\theta} \mathrm{s} \theta-\dot{u}_{2} \mathrm{c} \theta, \quad \dot{v}_{s y}=\ddot{y}-u_{2} \dot{\theta} \mathrm{c} \theta-\dot{u}_{2} \mathrm{~s} \theta .
$$

The theoretical formulas presented above cannot be practically utilized yet, since they involve particular velocity and acceleration components of the robot platform. In the experimental setup presented in this paper, they are estimated using the vision measurement system in cooperation with the set of three Kalman filters designed independently for every $i$-th component of the robot state $\left(q_{1}=\theta, q_{2}=x, q_{3}=y\right)$. The discrete-time process model and the output equation for the $i$-th filter are defined as follows:

$$
\begin{aligned}
\boldsymbol{z}_{i}(n+1) & =\boldsymbol{H} \boldsymbol{z}_{i}(n)+\boldsymbol{w}_{i}(n), \\
y(n) & =\boldsymbol{c}^{T} \boldsymbol{z}_{i}(n)+\varepsilon_{i}(n),
\end{aligned}
$$

where $\boldsymbol{w}_{i}=\mathcal{N}\left(\mathbf{0}, \boldsymbol{W}_{i}\right), \boldsymbol{W}_{i}=\operatorname{diag}\left\{\sigma_{i q}^{2}, \sigma_{i v}^{2}, \sigma_{i a}^{2}\right\}, \varepsilon_{i}=$ $\mathcal{N}\left(0, \sigma_{y i}^{2}\right)$ are uncorrelated white noises and

$$
\boldsymbol{z}_{i}=\left[\begin{array}{c}
z_{i q} \\
z_{i v} \\
z_{i a}
\end{array}\right], \quad \boldsymbol{H}=\left[\begin{array}{ccc}
1 & T_{s} & 0 \\
0 & 1 & T_{s} \\
0 & 0 & 1
\end{array}\right], \quad \boldsymbol{c}=\left[\begin{array}{l}
1 \\
0 \\
0
\end{array}\right],
$$

with $T_{s}$ being a sampling time and $z_{i q}, z_{i v}$ and $z_{i a}$ denoting displacement, velocity and acceleration of the $i$-th degree of freedom of the robot platform, respectively, taking $z_{1 q} \triangleq \theta, z_{2 q} \triangleq x$, and $z_{3 q} \triangleq y$. The state equation (27) describes the model of kinematic relations between the first two finite differences for the $i$-th robot state variable. The output model (28) indicates that the measured signals used in the error equation of the Kalman filter are particular posture coordinates of the robot platform obtained in our case from the vision system (and corrupted by the measurement noise $\varepsilon_{i}$ ). Practical implementation of filters is classical 7 with the prediction and correction stages yielding on the output the corrected $i$-th estimate $\hat{\boldsymbol{z}}_{i}(n)$ in every $n$-th discrete time instant.

Using Kalman filter estimates, discrete-time equations for estimation of skid-slip velocity and acceleration components were practically implemented as follows:

$$
\begin{aligned}
\hat{\omega}_{s} & :=\delta_{\omega}\left(\hat{z}_{1 v}-u_{1}^{-}\right), \\
\hat{v}_{s x} & :=\delta_{x}\left(\hat{z}_{2 v}-u_{2}^{-} \cos \hat{z}_{1 q}\right), \\
\hat{v}_{s y} & :=\delta_{y}\left(\hat{z}_{3 v}-u_{2}^{-} \sin \hat{z}_{1 q}\right),
\end{aligned}
$$

and

$$
\begin{aligned}
& \hat{\dot{v}}_{s x}:=\delta_{x}\left(\hat{z}_{2 a}+u_{2} \hat{z}_{1 v} \sin \hat{z}_{1 q}-\hat{\dot{u}}_{2}^{f} \cos \hat{z}_{1 q}\right), \\
& \hat{\dot{v}}_{s y}:=\delta_{y}\left(\hat{z}_{3 a}-u_{2} \hat{z}_{1 v} \cos \hat{z}_{1 q}-\hat{\dot{u}}_{2}^{f} \sin \hat{z}_{1 q}\right),
\end{aligned}
$$

\footnotetext{
${ }^{6}$ In general, also other sensor types like accelerometers or gyroscopes can be alternatively used.

${ }^{7}$ For implementation details, the reader is referred to (Bar-Shalom et al., 2001).
}

where $u_{1}^{-}$and $u_{2}^{-}$denote control signal samples delayed by one sample ${ }^{8}$. The term $\dot{u}_{2}$ was approximated bu $\hat{\dot{u}}_{2}^{f}$ as a filtered (by a first-order linear filter) finite difference of the control signal $u_{2}$. The quantities $\delta_{\omega}, \delta_{x}, \delta_{y} \in[0,1]$ have been introduced as the so-called cautious compensation coefficients. They turn out to be very important in practical implementation of the skid-slip compensator. Their usage is helpful in very demanding motion conditions arising during the tracking task, when the substantial wheel slip directly limits the possibility of control input influence on the robot motion. The effect of cross-coupling between the control action and the slip phenomenon was been modeled in the kinematic analysis conducted before. Therefore, cautious compensation coefficients provide a simple way of taking into account this cross-coupling effect.

In the conducted experiments, the estimates from the equations (29) - (33) were used in the control law (19)(20) instead of their particular theoretical counterparts.

4.3. Experimental results. Experimental tests were conducted using reference circular trajectory with radius $0.3 \mathrm{~m}$ and assuming constant reference linear and angular velocities $u_{2 t}(\tau)=0.93 \mathrm{~m} / \mathrm{s}$ and $u_{1 t}=3.1 \mathrm{rad} / \mathrm{s}$, respectively. The gains of the VFO controller were selected as 9 $k_{1}=2$ and $k_{p}=1$. The coefficients $\delta_{x}, \delta_{y}$ and $\delta_{\omega}$ used for cautious skid-slip compensation were chosen based on trials and errors as follows: $\delta_{x}=\delta_{y}=0.4$ and $\delta_{\omega}=0.45$.

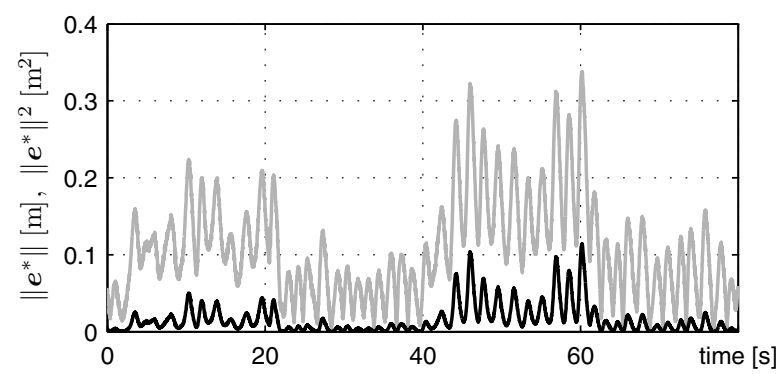

Fig. 4. Ex. A-Norms of position tracking errors: $\left\|e^{*}\right\|(\square)$, $\left\|e^{*}\right\|^{2}(\mathbf{\square})$.

The experiments were conducted using three different plane grounds: A-smooth teflon-like surface, Bporous veneered surface, and $\mathrm{C}$ - smooth varnished surface. For each experiment, during the first $10 \mathrm{~s}$ the skidslip compensator was switched OFF. At this stage, the initial tracking errors were bounded and persistent skidding conditions were obtained. Within the next $80 \mathrm{~s}$, the robot motion and control signals were recorded and the skid-slip

\footnotetext{
${ }^{8}$ To avoid algebraic loops in practical implementation, since skid-slip velocity estimates are needed in computations of 19 and 20 .

${ }^{9}$ Selected values of VFO gains result from arguments of closed-loop system stability, which is strongly influenced by the relatively low bandwidth of the vision feedback used in the experiments (details of VFO synthesis rules can be found in (Michałek and Kozłowski, 2009).
} 


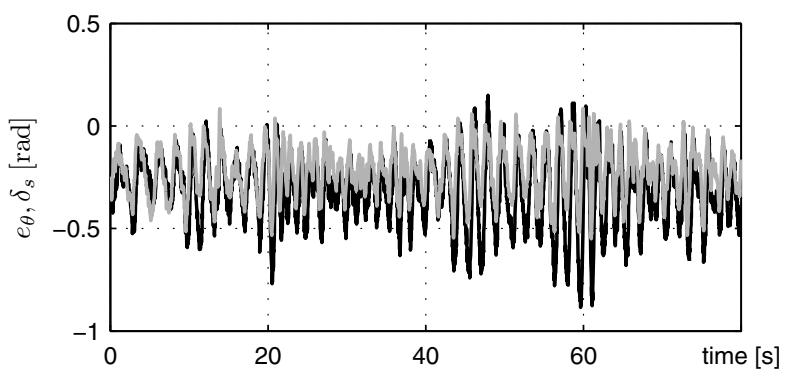

Fig. 5. Ex. A-Orientation tracking error $e_{\theta}(\mathbf{\square})$, skid-slip angle $\delta_{s}(\square)$

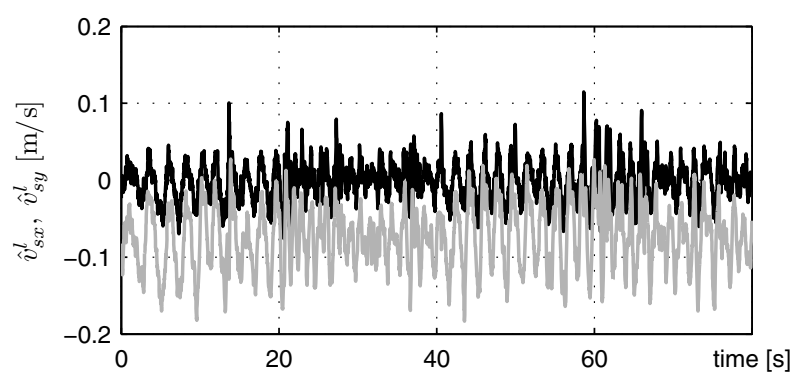

Fig. 6. Ex. A-Estimates of skid-slip velocity components expressed in the local frame: $\hat{v}_{s x}^{l}(\mathbb{\square}), \hat{v}_{s y}^{l}(\square)$.

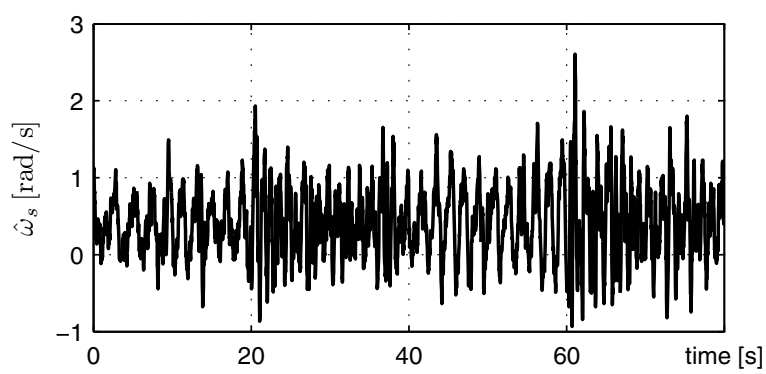

Fig. 7. Ex. A-Estimate of an angular slip.

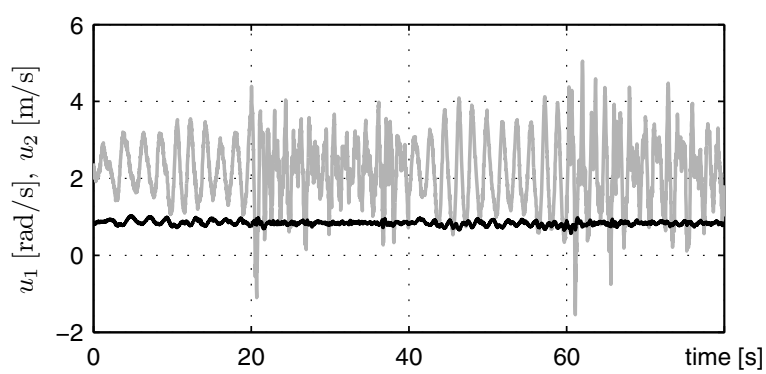

Fig. 8. Ex. A-Control signals: $u_{1}(\square), u_{2}$

compensator was toggled ON/OFF with the period of $20 \mathrm{~s}$ according to Table 1 . The experimental results obtained in permanent skid-slip motion conditions for the surfaces A, B and C are illustrated in Figs. 4, 8, 9,13 and 14, 18, respectively.

Figures 4,9 and 14 reveal the effectiveness of the VFO control strategy, where substantial decreasing of the position tracking error norm can be seen in the time inter-

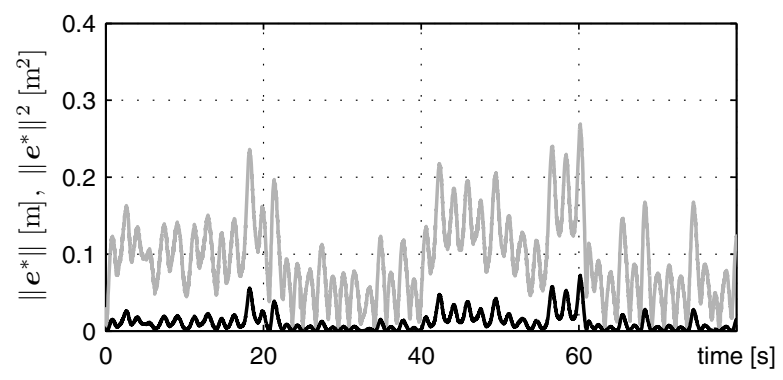

Fig. 9. Ex. B-Norms of position tracking errors: $\left\|e^{*}\right\|(\square)$, $\left\|e^{*}\right\|^{2}$

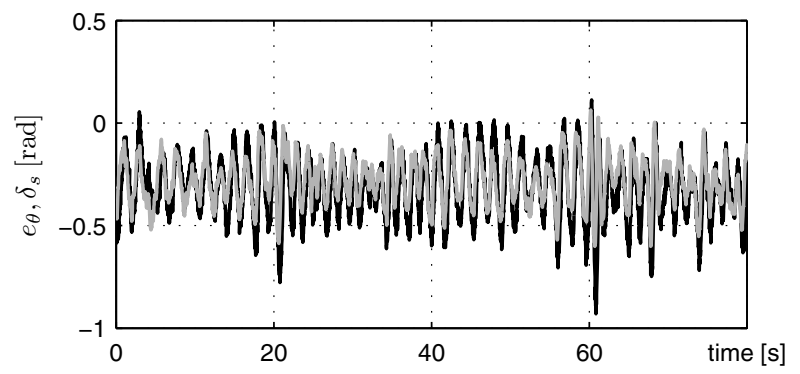

Fig. 10. Ex. B-Orientation tracking error $e_{\theta}(\mathbf{\square})$, skid-slip angle $\delta_{s}(\square)$.

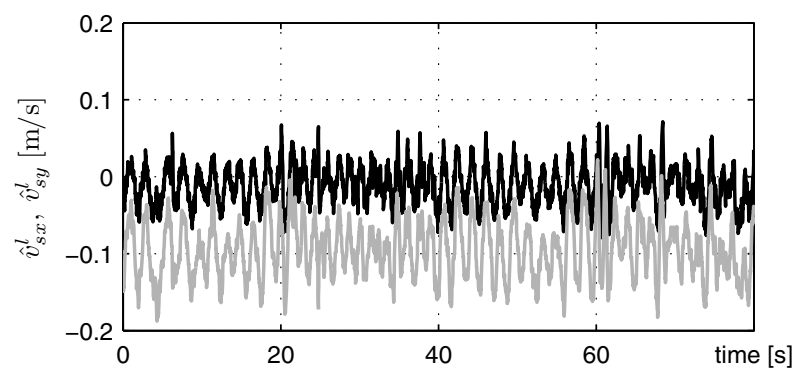

Fig. 11. Ex. B-Estimates of skid-slip velocity components expressed in the local frame: $\hat{v}_{s x}^{l}(\square), \hat{v}_{s y}^{l}(\square)$.

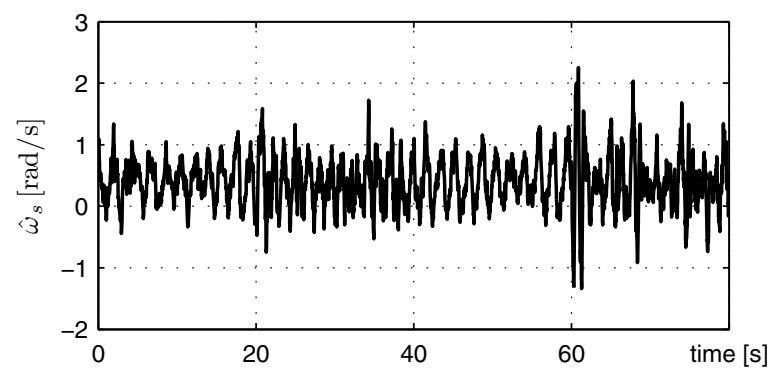

Fig. 12. Ex. B-Estimate of an angular slip.

vals when the skid-slip compensator is turned ON. Similar improvement results were obtained for all three types of slippery ground surfaces used during the tests. Moreover, they were obtained for the same set of controller parameters and the same values of cautious compensation coefficients. A slightly worse result can be seen for the case C (see Fig. 14), where the highly slippery surface caused 


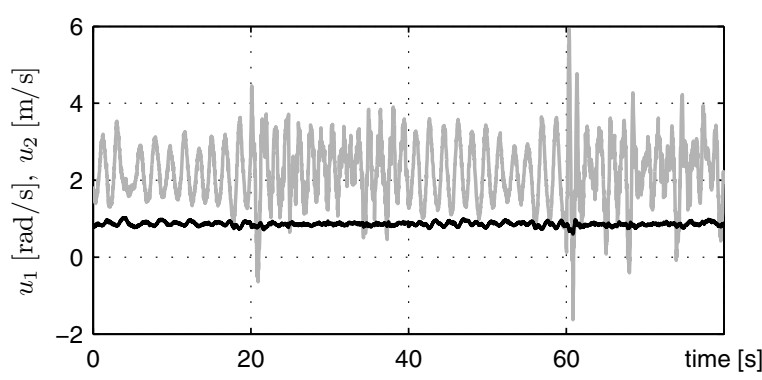

Fig. 13. Ex. B-Control signals: $u_{1}(\square), u_{2}(\mathbf{\square})$.

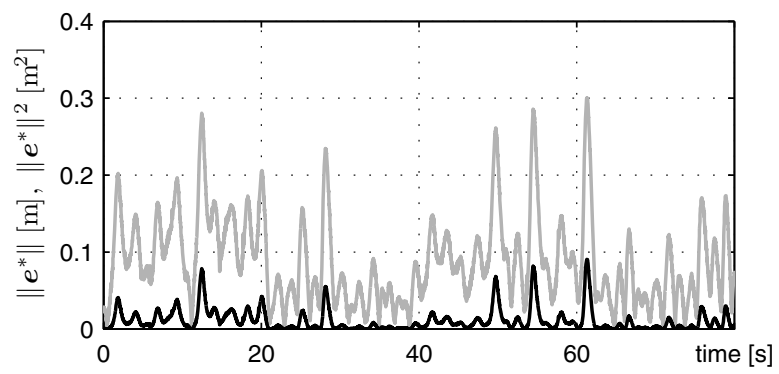

Fig. 14. Ex. C-Norms of position tracking errors: $\left\|e^{*}\right\|(\square)$, $\left\|e^{*}\right\|^{2}(\mathbf{\square})$

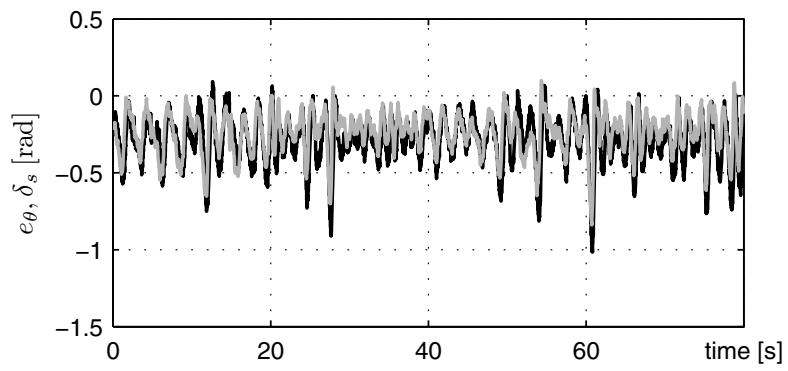

Fig. 15. Ex. C-Orientation tracking error $e_{\theta}(\mathbf{\square})$, skid-slip angle $\delta_{s}(\square)$.

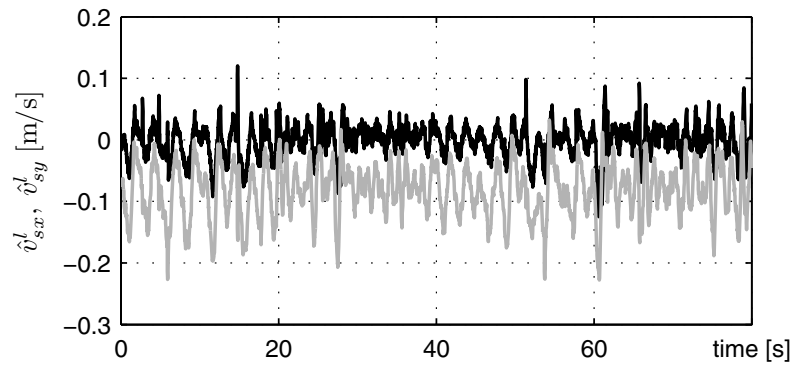

Fig. 16. Ex. C-Estimates of skid-slip velocity components expressed in the local frame: $\hat{v}_{s x}^{l}(\mathbf{\square}), \hat{v}_{s y}^{l}(\square)$.

that skid-slip phenomena were especially intensive (compare the skid-slip values in Fig. 16) with probably higher cross-coupling between the control action and the slip effect. The orientation error evolution illustrated in Figs. 5, 10 and 15 indicates quite a high but bounded level of values achieved by this signal (even $1 \mathrm{rad}$ ). The reason for high fluctuations of the orientation error is the fact that

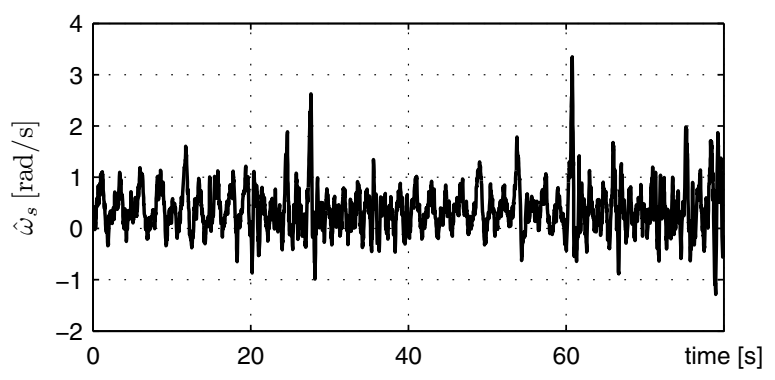

Fig. 17. Ex. C-Estimate of an angular slip.

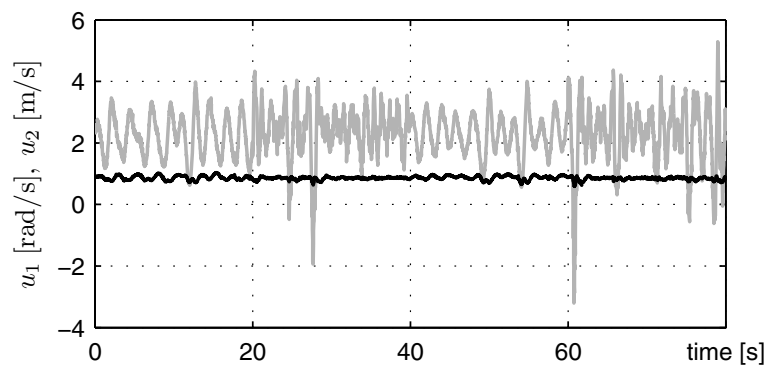

Fig. 18. Ex. C—Control signals: $u_{1}(\square), u_{2}(\square)$.

Table 1. State of the skid-slip compensator within subsequent time intervals during experiments.

\begin{tabular}{|c|c|}
\hline Time range $[\mathrm{s}]$ & Compensator state \\
\hline \hline$[0,20)$ & OFF \\
\hline$[20,40)$ & ON \\
\hline$[40,60)$ & OFF \\
\hline$[60,80)$ & ON \\
\hline
\end{tabular}

the lateral skid as an unmatched disturbance 10 cannot be directly compensated by control inputs like in the case of matched/input additive disturbances. Thus a substantial change of robot orientation is the way of compensating this type of hard disturbance. It is interesting to see that, when compensation is used $(\mathrm{ON})$, the fluctuation level of the orientation error value is usually considerably smaller. Worth noting is the coincidence of the orientation error with the skid-slip angle ploted in the figure.

The estimates of the driving skid-slip components presented in Figs. 6, 11 and 16 were expressed in the local frame attached to the robot body according to the following relationship: $\left[\hat{v}_{s x}^{l} \hat{v}_{s y}^{l}\right]^{T}=\boldsymbol{R}^{T}(\theta) \hat{\boldsymbol{v}}_{s}^{*}$, where $\boldsymbol{R}(\theta) \in \mathrm{SO} 2$ denotes a rotation operator on a plane. The results indicate that $\hat{v}_{s x}^{l}$ is not significant (at least in the sense of its mean value), whereas the skid component in the lateral direction $\hat{v}_{s y}^{l}$ is persistent and substantialabout $0.1 \mathrm{~m} / \mathrm{s}$ in the mean value, but temporarily even almost $0.2 \mathrm{~m} / \mathrm{s}$. Note that skid and slip phenomena are present during the OFF as well as ON intervals of motion.

\footnotetext{
${ }^{10}$ See (Wang and Low, 2008) for details.
} 
This results from the fact that the compensation scheme does not aim at attenuating skid-slip effects but only their influence on the robot motion.

According to Figs. 8, 13 and 18, one can notice that the most important role in the proposed motion control, especially when the compensator is switched ON, is played by the orienting control $u_{1}$. The pushing control $u_{2}$ changes only in a small range about its mean value, especially when the compensator is turned OFF.

Additional Figs. 19, 21 illustrate the position paths of the robot together with the reference trajectory path for a long-term vehicle motion in persistent skid-slip conditions with a fixed compensator state (ON or OFF) during the time interval of $80 \mathrm{~s}$. It is worth noting that the longdistance measure of the position difference between the reference path and the robot path seems to be considerably smaller for the case when skid-slip compensation is used. It is revealed by the more tight robot motion around the path of the reference trajectory. Let us recall, however, that this result should be treated rather as a positive sideeffect obtained during the experiments, since the motion control problem considered in the paper is the trajectory tracking task, which is more involved in practical realization. As a consequence, qualitative comparison of the geometrical results illustrated in Figs. 19, 21 with the time plots presented in Figs. 4 , 9 and 14 must be done with care, since time parametrization in the former has been lost.

\section{Concluding remarks}

In the paper, the VFO tracking controller with the skid-slip influence feed-forward compensation scheme was presented. The control strategy assumes treating skid and slip phenomena in a kinematic manner solely by their direct result in the form of an additional disturbing velocity vector. The kinematic approach allowed omitting hard modeling issues concerning the wheel-ground frictional interaction. An important part of the work was devoted to experimental validation of the proposed control concept. The results obtained on a laboratory setup equipped with the vision feedback and the Kalman filter estimator validated the effectiveness of the presented strategy, showing

- a visible and substantial improvement in position tracking accuracy in the case of compensator utilization,

- a similar tracking quality obtained for three different types of slippery ground surfaces for the same set of controller parameters,

- a positive side-effect of the more tight long-term vehicle movement around the path defined by the reference trajectory in the case of compensator usage.
The cost of the simplifying approach to the skid-slip treatment appeared as an unmodeled cross-coupling effect between the kinematic control action and the motion disturbance being compensated. This caused the necessity of introducing cautious compensation coefficients, which had to be properly tuned to guarantee tracking precision improvement together with the stability of the closed-loop system. Automatic (adaptive) tuning of these coefficients remains an open research problem, which can be taken into account in the near future.

\section{References}

Bar-Shalom, Y., Li, X. R. and Kirubarajan, T. (2001). Estimation with Applications to Tracking and Navigation, WileyInterscience, New York, NY.

Corradini, M. L., Leo, T. and Orlando, G. (1999). Robust stabilization of a mobile robot violating the nonholonomic constraint via quasi-sliding modes, Proceedings of the American Control Conference, San Diego, CA, USA, pp. 39353939.

Dixon, W. E., Dawson, D. M. and Zergeroglu, E. (2000). Tracking and regulation control of a mobile robot system with kinematic disturbances: A variable structure-like approach, Journal of Dynamic Systems, Measurement and Control 122(4): 616-623.

Fukao, T., Miyasaka, S., Mori, K., Adachi, N. and Osuka, K. (2001). Active steering systems based on model reference adaptive nonlinear control, Proceedings of the IEEE Intelligent Transportation Systems Conference, Oakland, CA, USA, pp. 502-507.

Khalil, H. K. (2002). Nonlinear Systems. 3rd Edn., PrenticeHall, Upper Saddle River, NJ.

Kiencke, U. and Nielsen, L. (2000). Automotive Control Systems, Springer-Verlag, Berlin.

Lenain, R., Thuilot, B., Cariou, C. and Martinet, P. (2006). High accuracy path tracking for vehicles in presence od sliding: Application to farm vehicle automatic guidance for agricultural tasks, Autonomous Robots 21(1): 79-97.

Leroquais, W. and dAndrea Novel, B. (1996). Modeling and control of wheeled mobile robots not satisfying ideal velocity constraints: the unicycle case, Proceedings of the 35th Conference on Decision and Control, Kobe, Japan, pp. 1437-1442.

Lewis, A. D. (1999). When is a mechanical control system kinematic? Proceedings of the 38th Conference on Decision and Control, Phoenix, AZ, USA, pp. 1162-1167.

Lhomme-Desages, D., Grand, C. and Guinot, J.-C. (2007). Trajectory control of a four-wheel skid-steering vehicle over soft terrain using physical interaction model, Proceedings of the IEEE International Conference on Robotics and Automation, Rome, Italy, pp. 1164-1169.

Mi, C., Lin, H. and Zhang, Y. (2005). Iterative learning control of antilock braking of electric and hybrid vehicles, IEEE Transactions on Vehicular Technology 54(2): 486-494. 

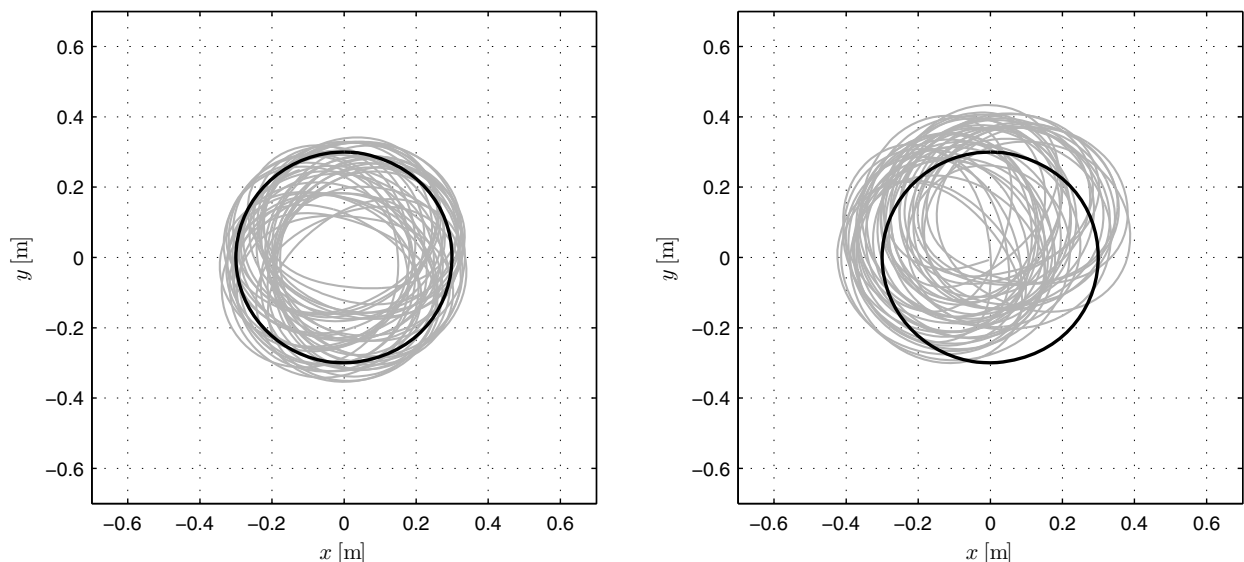

Fig. 19. Ground type A-Reference ( $\square$ ) and vehicle $(\square)$ position paths during long-term motion: compensator ON (left), compensator OFF (right).
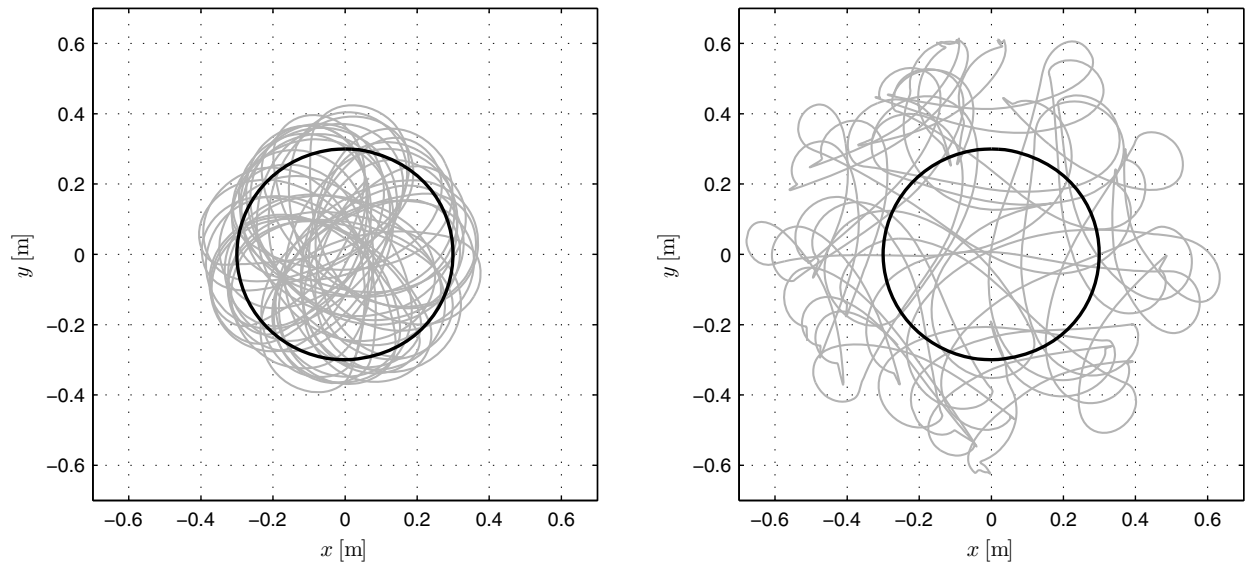

Fig. 20. Ground type B-Reference ( $\square$ ) and vehicle ( $\square$ ) position paths during long-term motion: compensator ON (left), compensator OFF (right).
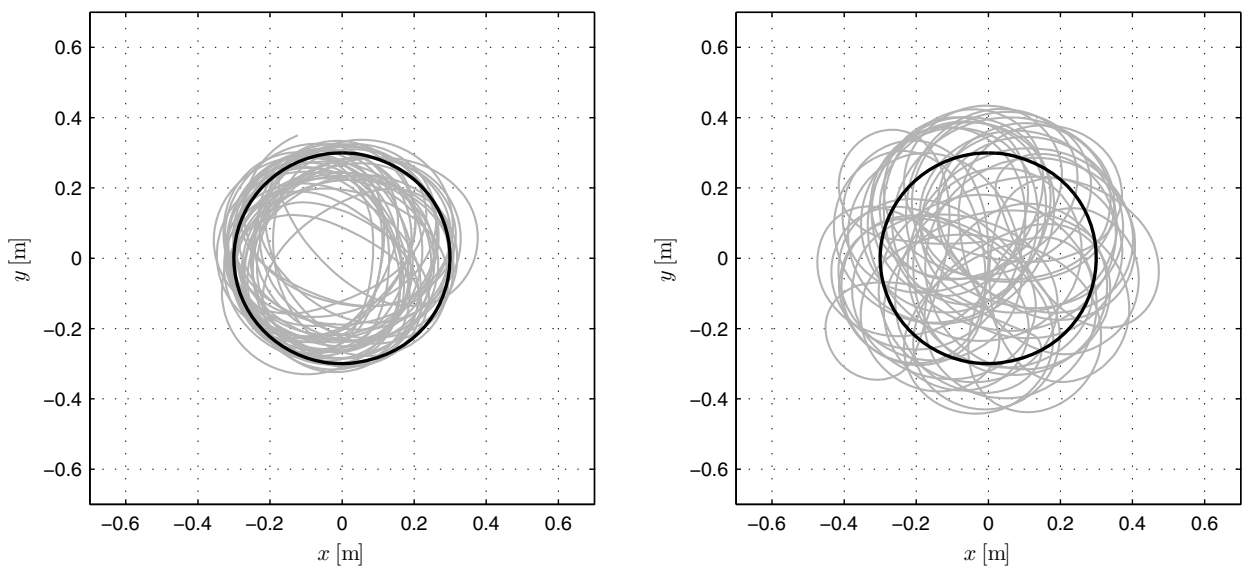

Fig. 21. Ground type C-Reference ( $\square$ ) and vehicle $(\square)$ position paths during long-term motion: compensator ON (left), compensator OFF (right).

Michałek, M. (2007). VFO control for mobile vehicles in the presence of skid phenomenon, Robot Motion and Control 2007, Lecture Notes in Control and Information Sciences, Vol. 360, Springer, pp. 57-66.
Michałek, M. and Kozłowski, K. (2009). Vector-field-orientation feedback control method for a differentially-driven vehicle, IEEE Transactions on Control Systems Technology, DOI: 10.1109/TCST.2008.2010406, (in print). 
Motte, I. and Campion, G. (2000). A slow manifold approach for the control of mobile robots not satisfying the kinematic constraints, IEEE Transactions on Robotics and Automation 16(6): 875-880.

Pacejka, H. B. (2002). Tyre and Vehicle Dynamics, ButterworthHeinemann.

Pazderski, D. and Kozłowski, K. (2008). Trajectory tracking control of skid-steering robot-Experimental validation, Proceedings of the 17th World Congress, International Federation of Automatic Control, Seoul, Korea, pp. 53775382.

Peng, S.-T., Sheu, J.-J. and Chang, C.-C. (2004). On one approach to constraining wheel slip for the autonomus control of a 4ws/4wd, Proceedings of the International Conference on Control Applications, Taipei, Taiwan, pp. 12541259.

Wang, D. and Low, C. B. (2008). Modeling and analysis of skidding and slipping in wheeled mobile robots: Control design perspective, IEEE Transactions on Robotics 24(3): 676-687.

Wong, J. Y. (2001). Theory of Ground Vehicles, John Wiley \& Sons, Inc., Ottawa.

Zong, Z., Zweiri, Y. H. and Seneviratne, L. D. (2006). Nonlinear observer for slip estimation of skid-steering vehicles, Proceedings of the IEEE International Conference on Robotics and Automation, Orlando, FL, USA, pp. 14991504.

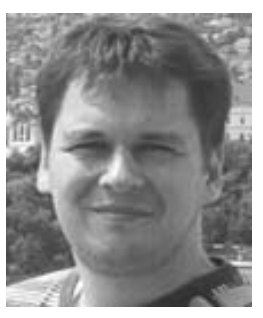

Maciej Michałek received the M.Sc. and the Ph.D. degree in automatics and robotics from the Poznań University of Technology (PUT), Poland, in 2001 and 2006, respectively. He is an assistant professor at the Chair of Control and Systems Engineering of the PUT, where he lectures and conducts research. His current research interests include control theory and applications for nonlinear, nonholonomic and robotic systems.

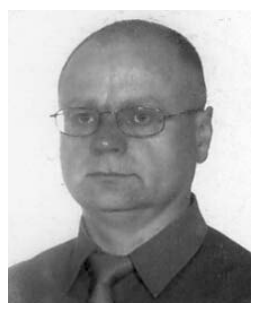

Piotr Dutkiewicz received the M.Sc. degree in electrical engineering from the Poznań University of Technology, Poland, in 1987, and the Ph.D. degree in control engineering and robotics in 1996 from the same university, where he is currently working toward the postdoctoral degree. His research interests include robot control, experimental parameter identification, sensor systems, and vision based control.

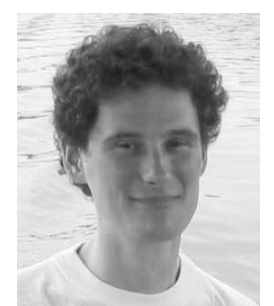

Marcin Kielczewski received the M.Sc. degree in automatics and robotics from the Poznań University of Technology (PUT), Poland, in 2000. He is an assistant lecturer at the Chair of Control and Systems Engineering of the PUT, where he lectures and conducts research. His current research interests include image processing and applications of vision systems in robotics.

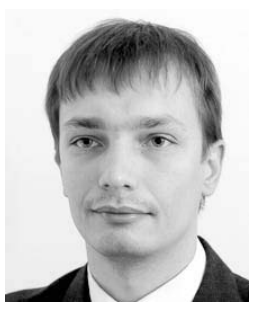

Dariusz Pazderski is with the Chair of Control and Systems Engineering of the Poznań University of Technology. He received his M.Sc degree in robotics in 2002 from the Faculty of Electrical Engineering and his Ph.D. degree in 2007 from the Faculty of Computing Science and Management of the Poznań University of Technology. His research interest include continuous and discontinuous nonlinear control, robust and adaptive control, motion planning, sensor systems, and applications of mobile robots.

\section{Appendix}

Derivation of the relations (24). Using the left-hand side equation from (23) together with (6) and (18) allows one to write

$$
\begin{aligned}
\boldsymbol{r} & =\boldsymbol{H}^{*}-\boldsymbol{g}_{2}^{*} u_{2}=\left[\begin{array}{l}
H_{2} \\
H_{3}
\end{array}\right]-\left[\begin{array}{l}
u_{2} \cos \theta \\
u_{2} \sin \theta
\end{array}\right] \\
& =\left\|\boldsymbol{H}^{*}\right\|\left[\begin{array}{c}
\frac{H_{2}}{\left\|\boldsymbol{H}^{*}\right\|}-\cos \alpha \cos \theta \\
\frac{H_{3}}{\left\|\boldsymbol{H}^{*}\right\|}-\cos \alpha \sin \theta
\end{array}\right] .
\end{aligned}
$$

Thus we obtain

$$
\begin{aligned}
\|\boldsymbol{r}\|^{2} & \\
= & \left\|\boldsymbol{H}^{*}\right\|^{2}\left[\frac{H_{2}^{2}}{\left\|\boldsymbol{H}^{*}\right\|^{2}}-\frac{2 H_{2} \cos \alpha \cos \theta}{\left\|\boldsymbol{H}^{*}\right\|}\right. \\
& +\cos ^{2} \alpha \cos ^{2} \theta \\
& \left.+\frac{H_{3}^{2}}{\left\|\boldsymbol{H}^{*}\right\|^{2}}-\frac{2 H_{3} \cos \alpha \sin \theta}{\left\|\boldsymbol{H}^{*}\right\|}+\cos ^{2} \alpha \sin ^{2} \theta\right] \\
= & \left\|\boldsymbol{H}^{*}\right\|^{2}\left[1-2 \cos \alpha \frac{H_{2} \cos \theta+H_{3} \sin \theta}{\left\|\boldsymbol{H}^{*}\right\|}+\cos ^{2} \alpha\right] \\
= & \left\|\boldsymbol{H}^{*}\right\|^{2}\left(1-2 \cos \alpha \cos \alpha+\cos ^{2} \alpha\right) \\
= & \left\|\boldsymbol{H}^{*}\right\|^{2}\left(1-\cos ^{2} \alpha\right)
\end{aligned}
$$

and, finally, $\|\boldsymbol{r}\|=\left\|\boldsymbol{H}^{*}\right\| \sqrt{1-\cos ^{2} \alpha}=\left\|\boldsymbol{H}^{*}\right\| \gamma$.

The limit from the right-hand side of (24) can be explained using (6) and recalling that $\cos \alpha=$ $\left(\boldsymbol{g}_{2}^{* T} \boldsymbol{H}^{*}\right) /\left(\left\|\boldsymbol{g}_{2}^{*}\right\|\left\|\boldsymbol{H}^{*}\right\|\right)$ :

$$
\begin{aligned}
\gamma^{2}(\theta) & =1-\cos ^{2} \alpha(\theta)=1-\frac{\left(H_{2} \cos \theta+H_{3} \sin \theta\right)^{2}}{\left\|\boldsymbol{H}^{*}\right\|^{2}\left\|\boldsymbol{g}_{2}^{*}(\theta)\right\|^{2}} \\
& =\frac{H_{2}^{2}+H_{3}^{2}-\left(H_{2} \cos \theta+H_{3} \sin \theta\right)^{2}}{H_{2}^{2}+H_{3}^{2}} \\
& =\frac{\left(H_{2} \sin \theta-H_{3} \cos \theta\right)^{2}}{H_{2}^{2}+H_{3}^{2}} .
\end{aligned}
$$

The definition (13) allows one to write

$$
\tan \theta \stackrel{\theta \rightarrow \theta_{a}}{\longrightarrow} \frac{H_{3}}{H_{2}} \Rightarrow \sin \theta \stackrel{\theta \rightarrow \theta_{a}}{\longrightarrow} \frac{H_{3} \cos \theta}{H_{2}},
$$

which, after substitution into the preceding equation, gives the conclusion $\lim _{\theta \rightarrow \theta_{a}} \gamma(\theta)=0$. 
Uniform continuity and integrability of the function

(25). Let us first check uniform continuity of $W(\tau)$. From (25) one can see that $W$ is continuous since all the terms on the right-hand side are continuous. The timederivative of $W(\tau)$ can be computed as follows:

$$
\begin{aligned}
\dot{W}= & -\dot{\gamma}\left(k_{p}\left\|\boldsymbol{e}^{*}\right\|^{2}+\kappa\left\|\boldsymbol{e}^{*}\right\|\right) \\
& +2 k_{p}(1-\gamma)\left\|\boldsymbol{e}^{*}\right\| \frac{\mathrm{d}\left\|\boldsymbol{e}^{*}\right\|}{\mathrm{d} \tau} \\
& -\gamma\left(\frac{\mathrm{d}\left\|\boldsymbol{e}^{*}\right\|}{\mathrm{d} \tau} \kappa+\left\|\boldsymbol{e}^{*}\right\| \dot{\kappa}\right),
\end{aligned}
$$

where

$$
\begin{gathered}
\frac{\mathrm{d}\left\|\boldsymbol{e}^{*}\right\|}{\mathrm{d} \tau}=\frac{\boldsymbol{e}^{* T} \dot{\boldsymbol{e}}^{*}}{\left\|\boldsymbol{e}^{*}\right\|}, \quad \dot{\kappa}=\frac{\mathrm{d}\left\|\boldsymbol{q}_{t}^{*}\right\|}{\mathrm{d} \tau}+\frac{\mathrm{d}\left\|\boldsymbol{v}_{s g}^{*}\right\|}{\mathrm{d} \tau}, \\
\dot{\gamma}=\frac{A\left\|\boldsymbol{H}^{*}\right\|^{2}-\left(H_{2} \mathrm{~s} \theta-H_{3} \mathrm{c} \theta\right)\left(H_{2} \dot{H}_{2}+H_{3} \dot{H}_{3}\right)}{\left\|\boldsymbol{H}^{*}\right\|^{3}}, \\
A=\left(\dot{H}_{2} \mathrm{~s} \theta+H_{2} \dot{\theta} \mathrm{c} \theta-\dot{H}_{3} \mathrm{c} \theta+H_{3} \dot{\theta} \mathrm{s} \theta\right), \\
\dot{\boldsymbol{H}}^{*}=\left[\begin{array}{ll}
\dot{H}_{2} & \dot{H}_{3}
\end{array}\right]^{T}=k_{p} \dot{\boldsymbol{e}}^{*}+\ddot{\boldsymbol{q}}_{t}^{*}-\dot{\boldsymbol{v}}_{s}^{*}
\end{gathered}
$$

with the notation $\mathrm{s} \theta \equiv \sin \theta, \mathrm{c} \theta \equiv \cos \theta$, used for compactness. Recalling now the assumptions made in Proposition 1 together with A1-A2 and since $\left|\mathrm{d}\left\|e^{*}\right\| / \mathrm{d} \tau\right| \leqslant$ $\left\|\dot{e}^{*}\right\|$ with $\left\|\dot{e}^{*}\right\| \in \mathcal{L}_{\infty}$ (see the Proof), one can conclude that the time derivative (5) is bounded and, consequently, the function $W(\tau)$ is uniformly continuous.

Let us now consider the integrability of the function 25) introducing the following decomposition: $W(\tau)=$ $w_{1}(\tau)+w_{2}(\tau)$, where

$$
\begin{aligned}
& w_{1}(\tau)=k_{p}(1-\gamma(\tau))\left\|\boldsymbol{e}^{*}(\tau)\right\|^{2} \geqslant 0, \\
& w_{2}(\tau)=-\gamma(\tau)\left\|\boldsymbol{e}^{*}(\tau)\right\| \kappa(\tau) \leqslant 0 .
\end{aligned}
$$

We will analyze the integrability of $w_{1}(\tau)$ and $w_{2}(\tau)$. For this purpose, let us introduce an upper bound of $\kappa(\tau)$ :

$$
K \triangleq \sup _{\tau \geqslant 0} \kappa(\tau)=\sup _{\tau \geqslant 0}\left(\left\|\dot{\boldsymbol{q}}_{t}^{*}(\tau)\right\|+\left\|\boldsymbol{v}_{s}^{*}(\tau)\right\|\right)<\infty,
$$

which exists by assumption, and an upper bound

$$
E \triangleq \sup _{\tau \geqslant 0}\left\|e^{*}(\tau)\right\|<\infty
$$

which exists according to the analysis conducted in the proof. Using the above bounds, one obtains

$$
\int_{0}^{\infty}-w_{2}(\tau) \mathrm{d} \tau \leqslant K E \int_{0}^{\infty} \gamma(\tau) \mathrm{d} \tau
$$

Let us assess the upper bound of the integral $\int_{0}^{\infty} \gamma(\tau) \mathrm{d} \tau$ as follows: According to the notation introduced in the proof, $\gamma(\tau)=\sqrt{1-\cos ^{2} \alpha(\tau)}=$ $|\sin \alpha(\tau)| \in[0,1]$. Using (13), (14) and since $\alpha(\tau)=\angle\left(\boldsymbol{g}_{2}^{*}(\theta(\tau)), \boldsymbol{H}^{*}(\tau)\right)$, one can write $|\sin \alpha(\tau)|=$ $\left|\sin \left(e_{a}(\tau) \pm N \pi\right)\right|$, where $N=0$ for $u_{2 t}(\tau)>0$ and $N=1$ for $u_{2 t}(\tau)<0$ (note that $N$ is constant for the whole control time-horizon, since $u_{2 t}(\tau)$ cannot cross zero for all $\tau \geqslant 0$ due to the persistent excitation condition-see Section 2). Thus, for absolute values, one obtains

$$
\begin{aligned}
\int_{0}^{\infty} \gamma(\tau) \mathrm{d} \tau & =\int_{0}^{\infty}|\sin \alpha(\tau)| \mathrm{d} \tau \\
& =\int_{0}^{\infty}\left|\sin e_{a}(\tau)\right| \mathrm{d} \tau \\
& \leqslant \int_{0}^{\infty}\left|e_{a}(\tau)\right| \mathrm{d} \tau \\
& =\int_{0}^{\infty}\left|e_{a}(0)\right| \exp \left(-k_{1} \tau\right) \mathrm{d} \tau=\frac{\left|e_{a}(0)\right|}{k_{1}}
\end{aligned}
$$

where the exponential convergence result from (21) has been utilized. Since $\left|e_{a}(0)\right|<\infty$ (by assumption) and $k_{1}>0$, one concludes that the integral $\int_{0}^{\infty} \gamma(\tau) \mathrm{d} \tau=G$ is finite. Proceeding the estimation for (37), one obtains

$$
0 \leqslant \int_{0}^{\infty}-w_{2}(\tau) \mathrm{d} \tau=W_{2} \leqslant K E G<\infty,
$$

which allows concluding about the integrability of $w_{2}(\tau)$ in $[0, \infty)$.

Next, we utilize the result from the proof where we obtained $\dot{V}(\tau) \leqslant-W(\tau)$, which can be written as $\dot{V}(\tau) \leqslant-\left(w_{1}(\tau)+w_{2}(\tau)\right)$. Integrating both sides of the latter inequality gives

$$
\int_{0}^{\infty} w_{1}(\tau) \mathrm{d} \tau \leqslant-\int_{0}^{\infty} \dot{V}(\tau) \mathrm{d} \tau+\int_{0}^{\infty}-w_{2}(\tau) \mathrm{d} \tau
$$

and, further,

$$
\begin{aligned}
W_{1}=\int_{0}^{\infty} w_{1}(\tau) \mathrm{d} \tau & \leqslant V(0)-V(\infty)+W_{2} \\
& \leqslant V(0)+K E G<\infty
\end{aligned}
$$

with (38) and the non-negativeness of $w_{1}(\tau)$ and $V(\infty)$ utilized. Finally, due to (38) and (39) we can conclude what follows:

$$
\begin{aligned}
\int_{0}^{\infty} W(\tau) \mathrm{d} \tau & =\int_{0}^{\infty} w_{1}(\tau) \mathrm{d} \tau+\int_{0}^{\infty} w_{2}(\tau) \mathrm{d} \tau \\
& =W_{1}-W_{2}<\infty
\end{aligned}
$$

The above inequality implies the integrability of the function $W(\tau)$.

Barbalat's lemma (according to (Khalil, 2002)). Let $\phi: \mathbb{R} \mapsto \mathbb{R}$ be a uniformly continuous function on $[0, \infty)$. Suppose that $\lim _{\tau \rightarrow \infty} \int_{0}^{\tau} \phi(\xi) \mathrm{d} \xi$ exists and is finite. Then $\phi(\tau) \rightarrow 0$ as $\tau \rightarrow \infty$.

Received: 16 December 2008 Revised: 13 July 2009 